\title{
Erratum to: Improvement in outcomes after implantation of a novel polyurethane meniscal scaffold for the treatment of medial meniscus deficiency
}

\author{
Karl F. Schüttler · Steffen Pöttgen • Alan Getgood • \\ Marga B. Rominger · Susanne Fuchs-Winkelmann • \\ Philip P. Roessler $\cdot$ Ewgeni Ziring $\cdot$ Turgay Efe
}

Published online: 27 August 2014

(C) Springer-Verlag Berlin Heidelberg 2014

\section{Erratum to: Knee Surg Sports Traumatol Arthrosc}

DOI 10.1007/s00167-014-2977-6

We regret the erroneous Table 1 caption in the official publication. Also, Table 1 last column head should be P (pre OP versus 24 months and not 12 months).

The correct Table 1 and its caption are given below.

Table 1 Summary of patient-reported outcome at preoperative baseline, 6, 12 and 24 months post-surgery

\begin{tabular}{llllllll}
\hline KOOS & Preoperative & 6 months & $\begin{array}{l}\text { P (pre OP versus } \\
6 \text { months) }\end{array}$ & 12 months & $\begin{array}{l}\text { P (pre OP versus } \\
12 \text { months) }\end{array}$ & $\begin{array}{l}24 \text { months } \\
\text { P (pre OP versus } \\
24 \text { months) }\end{array}$ \\
\hline Pain & $47 \pm 14.5$ & $75 \pm 17.7$ & $p<0.0001$ & $82 \pm 17.4$ & $p<0.0001$ & $83 \pm 18.6$ & $p<0.0001$ \\
Symptom & $60 \pm 16.2$ & $67 \pm 18.5$ & $p=0.0091$ & $85 \pm 9.7$ & $p<0.0001$ & $81 \pm 13.4$ & $p=0.0003$ \\
ADL & $53 \pm 16.0$ & $85 \pm 14.5$ & $p<0.0001$ & $88 \pm 13.0$ & $p<0.0001$ & $91 \pm 14.7$ & $p<0.0001$ \\
Sport/Rec & $26 \pm 20.5$ & $60 \pm 25.3$ & $p=0.0001$ & $68 \pm 24.0$ & $p<0.0001$ & $66 \pm 28.5$ & $p<0.0001$ \\
QOL & $28 \pm 16.6$ & $55 \pm 26.9$ & $p=0.0007$ & $67 \pm 20.4$ & $p<0.0001$ & $63 \pm 18.9$ & $p<0.0001$ \\
KSS & & & & & & $96 \pm 7.9$ & $p<0.0001$ \\
Function score & $61 \pm 22.2$ & $87 \pm 10.2$ & $p=0.0001$ & $89 \pm 15.7$ & $p=0.0001$ & $88 \pm 12.4$ & $p<0.0001$ \\
Knee score & $65 \pm 9.4$ & $89 \pm 13.1$ & $p<0.0001$ & $87 \pm 14.1$ & $p<0.0001$ & $7.3 \pm 1.8$ & $p=0.0035$ \\
UCLA & $5.4 \pm 1.8$ & $6.1 \pm 1.8$ & n.s. & $6.5 \pm 2.1$ & n.s. & $1.5 \pm 2.1$ & $p<0.0001$ \\
VAS & $5.1 \pm 2.0$ & $2.1 \pm 2.4$ & $p=0.0004$ & $1.8 \pm 2.3$ & $p<0.0001$ & 9601 \\
\hline
\end{tabular}

The online version of the original article can be found under doi:10.1007/s00167-014-2977-6.

K. F. Schüttler $\cdot$ S. Pöttgen $\cdot$ S. Fuchs-Winkelmann ·

P. P. Roessler · T. Efe $(\square)$

Department of Orthopedics and Rheumatology, University

Hospital Marburg, Baldingerstrasse, 35043 Marburg, Germany

e-mail: efet@med.uni-marburg.de

A. Getgood

Fowler Kennedy Sport Medicine Clinic, University of Western

Ontario, London, ON, Canada
M. B. Rominger

Department of Radiology, University Hospital Marburg,

Marburg, Germany

\section{E. Ziring}

Department of Trauma, Hand and Reconstructive Surgery,

University Hospital Marburg, Marburg, Germany 